\title{
Body Cavity-Based Lymphoma in a Country with Low Human Immunodeficiency Virus Prevalence: A Series of 17 Cases from the Consortium for Improving Survival of Lymphoma
}

\author{
Junghoon Shin, MD \\ Young Hyeh Ko, MD, PhD² \\ Sung Yong $\mathrm{Oh}, \mathrm{MD}, \mathrm{PhD}^{3}$ \\ Dok Hyun Yoon, MD, PhD ${ }^{4}$ \\ Jeong-0k Lee, $M D, P h D^{5}$ \\ Jin Seok Kim, MD, PhD ${ }^{6}$ \\ Yong Park, $\mathrm{MD}, \mathrm{PhD}^{7}$ \\ Ho Jin Shin, MD, PhD \\ Seok Jin Kim, MD, $P h D^{9}$ \\ Jong Ho Won, MD, PhD'10 \\ Sung-Soo Yoon, MD, PhD' \\ Won Seog Kim, MD, PhD9 \\ Youngil Koh, MD, PhD 1 \\ On behalf of the Consortium for Improving \\ Survival of Lymphoma investigators
}

*A list of author's affiliations appears at the end of the paper.

\begin{abstract}
Purpose
Primary effusion lymphoma (PEL) is a type of body cavity-based lymphoma (BCBL). Most patients with PEL are severely immunocompromised and seropositive for human immunodeficiency virus (HIV). We investigated the distinctive clinicopathologic characteristics of $\mathrm{BCBL}$ in a country with low HIV burden.
\end{abstract}

\section{Materials and Methods}

We retrospectively collected data on the clinicopathologic characteristics, treatments, and outcomes of 17 consecutive patients with BCBL at nine institutions in Korea.

\section{Results}

Latency-associated nuclear antigen 1 (LANA1) immunostaining indicated that six patients had PEL, six patients had human herpesvirus 8 (HHV8)-unrelated BCBL, and five patients had HHV8-unknown BCBL. The patients with PEL exhibited no evidence of immunodeficiency except for one who was HIV positive. One (20\%) and four (80\%) patients with PEL and six (100\%) and zero (0\%) patients with HHV8-unrelated BCBL were positive for CD2O and CD30 expression, respectively. The two patients with PEL (one HIV-positive and one HIV-negative patient) with the lowest proliferation activity as assessed by the Ki-67 labeling index survived for $>1$ and $>4$ years without chemotherapy, respectively, in contrast to the PEL cases in the literature, which mostly showed a high proliferation index and poor survival.

\section{Conclusion}

PEL mostly occurred in ostensibly immunocompetent individuals and had a favorable outcome in Korea. A watchful waiting approach may be applicable for managing HIV-seronegative patients with PEL with a low Ki-67 labeling index. A possible trend was detected among LANA1, CD20, and CD30 expression in BCBL.
Correspondence: Won Seog Kim, MD, PhD Department of Medicine, Samsung Medical Center, 81 Irwon-ro, Gangnam-gu,

Seoul 06351, Korea

Tel: 82-2-3410-6548

Fax: 82-2-3410-1754

E-mail:wskimsmc@skku.edu

Co-correspondence: Youngil Koh, MD, PhD Department of Internal Medicine,

Seoul National University Hospital,

101 Daehak-ro, Jongno-gu, Seoul 03080, Korea

Tel: 82-2-2072-7217

Fax: 82-2-2072-7379

E-mail: go01@snu.ac.kr

Received October 8, 2018

Accepted January 28, 2019

Published Online February 14, 2019

\section{Key words}

Body cavity-based lymphoma, Primary effusion lymphoma, Human herpesvirus 8, Human immunodeficiency virus, Immunophenotype 


\section{Introduction}

Primary effusion lymphoma (PEL) is a rare subtype of nonHodgkin lymphoma (NHL) characterized by lymphomatous effusions in body cavities with or without extracavitary tumor formation [1]. Following the discovery of its universal association with human herpesvirus 8 (HHV8) infection [2], PEL was established as a distinct clinical entity and incorporated into the World Health Organization (WHO) classification system for lymphoid neoplasms [3,4]. PEL mostly occurs in patients with human immunodeficiency virus (HIV) infection [2,4]. In the four largest published series of PEL cases, each including 11-34 patients with classic (body cavitybased) PEL, more than $90 \%$ of the patients were youngto-middle-aged males who had HIV risk factors such as homosexuality or intravenous drug abuse [5-8]. Although non-HIV-associated PEL has occasionally been observed, the patients were either elderly ( $\geq 78$ years of age) or recipients of an organ transplant, or the disease developed in an artificial cavity related to a breast implant capsule, suggesting that systemic or local immunocompromising conditions were involved in the lymphomagenesis [4,9-11]. On the basis of those observations, it is considered unusual for PEL to occur in immunocompetent individuals.

Since the establishment of PEL as a subcategory of NHL, there have been numerous reports of HHV8-unrelated body cavity-based lymphoma (BCBL) [12-18], a provisional entity that does not fulfill the diagnostic criteria of PEL because of the absence of HHV8 infection in neoplastic cells [3]. HHV8unrelated BCBL has a remarkable clinicopathologic similarity to PEL in terms of its effusion-based growth and its large, pleomorphic, immunoblastic-to-anaplastic cytomorphology [17,18]; however, it differs from PEL in important aspects: (1) it commonly affects immunocompetent individuals and females; (2) the tumor cells often express pan-B-cell markers including CD19, CD20, and CD79a; and (3) the combination chemotherapy with or without rituximab shows promising efficacy, resulting in a better prognosis compared with that of PEL [16-18]. Therefore, it is important to differentiate between HHV8-unrelated BCBL and PEL to ensure proper management and prognostication of patients.

Because HIV/acquired immunodeficiency syndrome (AIDS) confers significant morbidity and mortality due to opportunistic infections and various malignancies, HIVunrelated PEL may exhibit distinctive clinical features and have a different prognosis compared with typical HIVrelated PEL. Most, if not all, patients with PEL have been described in North America and Europe [4-8], whereas around $60 \%$ of patients described with HHV8-unrelated BCBL are Japanese [16-18]. That remarkable imbalance in case distribution is in line with the low prevalence of HIV/
AIDS, the strongest risk factor for PEL, in eastern Asia [19]. Given the rarity of HIV / AIDS in eastern Asia, studies of PEL in patients from that area may provide novel insights into the nature of the disease.

Here we describe the clinicopathologic characteristics and outcomes of a series of 17 patients with BCBL in South Korea. The aim of this study was to identify the distinctive clinicopathologic characteristics and outcomes of BCBL in a country with low HIV burden. To our knowledge, this series represents the largest collection of novel cases of HIV-unrelated PEL and the largest Asian BCBL cohort that is independent of the existing literature.

\section{Materials and Methods}

\section{Case selection}

This retrospective study included all consecutive patients diagnosed with BCBL from April 2002 to October 2016 at nine institutions in South Korea. We defined BCBL as NHL that (1) presented exclusively or predominantly as a malignant effusion with or without extra-cavitary lesions, (2) was confirmed either by cytological evaluation of the malignant effusion or by tissue biopsy that revealed cytomorphology characteristic of PEL, and (3) did not meet the diagnostic criteria for any subtype of NHL other than PEL as per the current WHO classification on the basis of the clinical history, cytomorphology, immunophenotype, and genetic alterations (e.g., c-myc gene rearrangement in Burkitt lymphoma). We excluded patients diagnosed with Burkitt or Burkitt-like lymphoma, plasmablastic lymphoma, anaplastic large cell lymphoma, and pyothorax-associated lymphoma (designated as 'diffuse large B cell lymphoma associated with chronic inflammation' in the 2016 WHO classification) [3].

\section{Data collection}

We collected the following data for each patient via medical records review: demographics; sites of involved body cavities and extra-cavitary lesions; performance status; international prognostic index score; medical histories including hepatitis $B$ virus (HBV), hepatitis $\mathrm{C}$ virus (HCV), and HIV infections, co-occurring malignancies (e.g., Kaposi's sarcoma), multicentric Castleman disease, autoimmune disease, and iatrogenic immunosuppression such as organ transplantation, immunosuppressant administration, or cytotoxic chemotherapy; initial laboratory profiles; treatment history; and outcome. We reviewed pathology reports for all of the patients to confirm the diagnosis of BCBL and to obtain 


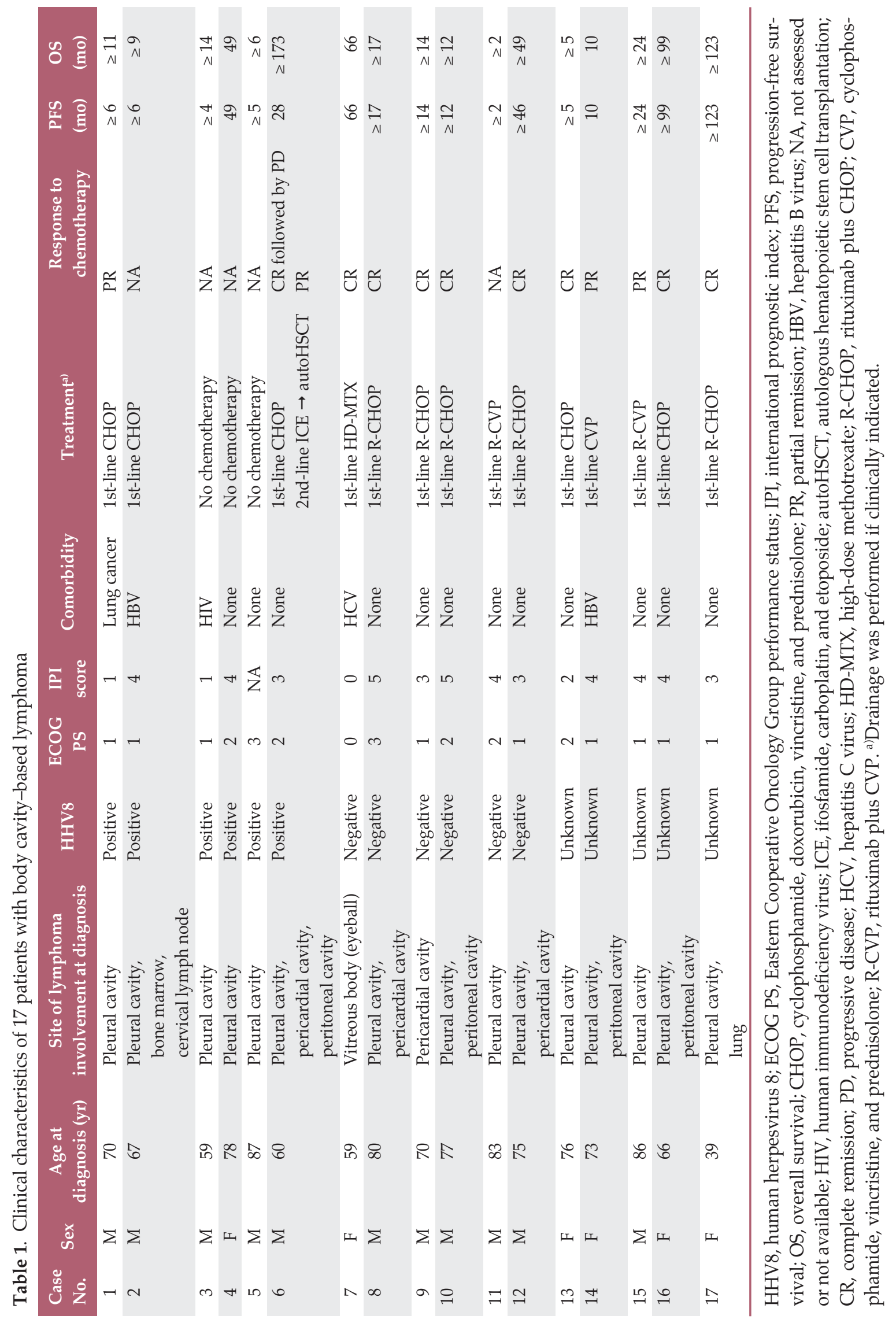


immunophenotype information. The HHV8 infection status of lymphoma cells was determined by immunocytochemical staining for latency-associated nuclear antigen 1 (LANA1) using mouse anti-HHV8 monoclonal antibodies (Cell Marq, Hot Springs, AR) and the Ventana BenchMark XT autostainer (Ventana Medical Systems, Tucson, AZ). Coinfection with Epstein-Barr virus (EBV) was assessed by in situ hybridization for EBV-encoded small RNA (EBER).

\section{Statistical analysis}

We divided the patients into three groups on the basis of the LANA1 immunostaining results-those with PEL (LANA1 positive), those with HHV8-unrelated BCBL (LANA1 negative), and those with HHV8-unknown BCBL (not tested for LANA1 expression)—-to describe and compare their clinicopathologic characteristics and prognoses. We summarized the data as medians (ranges) for continuous variables and numbers (\%) for discrete variables. We used the International Working Group response criteria to assess the response to treatment [20]. We defined progression-free survival (PFS) as the time from diagnosis to disease progression or death from any cause, whichever occurred first. To estimate PFS, we censored patients who were progression-free and alive at the time of the last assessment of the disease state. We defined overall survival (OS) as the time from diag-nosis to death from any cause. To estimate OS, we censored patients who were alive at the last follow-up date. We used the Kaplan-Meier method to estimate the median PFS and OS. No statistical test was performed because of the small number of cases.

\section{Ethical statement}

The Institutional Review Board at each participating institution reviewed and approved the study protocol (Seoul National University Hospital Institutional Review Board approval number: H-1511-051-718). Patient consent was waived because of the retrospective nature of the study and lack of patient interaction. We carried out all study procedures including data collection and analyses in accordance with the ethical standards of the Helsinki Declaration (revised in 2013; World Medical Association).

\section{Results}

\section{Clinical characteristics}

We identified a total of 17 BCBL cases. LANA1 immunostaining results were available for 12 patients, of which six were positive for LANA1 (indicating PEL) and six were negative for LANA1 (indicating HHV8-unrelated BCBL). The demographic and clinical characteristics of the patients are described in Table 1 and summarized for the entire cohort and for subgroups based on HHV8 positivity in Table 2. All of the patients were native Koreans of the Asian race. Diagnosis of BCBL was made by cytological evaluation of lymphomatous effusion in 12 patients $(70.6 \%)$ and by tissue biopsy in the remaining five patients (29.4\%) - pleural biopsy in four patients and cervical lymph node biopsy in one patient. At the time of diagnosis, four patients with PEL and one patient with HHV8-unrelated BCBL had B symptoms (fever, weight loss, or night sweats). The male-to-female ratio was 5:1 in both the PEL cohort and the HHV8-unrelated BCBL cohort and 1:4 in the HHV8-unknown BCBL cohort. Except in two patients (one with PEL who had cervical lymph node and bone marrow lesions and one with HHV8-unknown BCBL who had a lung lesion, all of which were pathologically confirmed), BCBL presented exclusively as malignant effusions involving one or multiple body cavities (Tables 1 and 2). The pleural space was the most frequently involved body cavity in all three categories of BCBL. Interestingly, one case of HHV8-unrelated BCBL exclusively involved the vitreous body of the eye (case 7) (Table 1).

Only one of the 17 patients with BCBL had HIV infection (case 3) (Table 1). Except for that patient, no patient had any apparent history of immunocompromising conditions or iatrogenic immunosuppression. One patient with PEL and one patient with HHV8-unknown BCBL were positive for HBV surface antigen. One patient with HHV8-unrelated BCBL was positive for anti-HCV antibody; however, a reverse transcription-polymerase chain reaction assay did not detect HCV RNA, indicating that the HCV infection was resolved. None of the patients had any history of Kaposi's sarcoma, multicentric Castleman disease, or autoimmune disease. Blood cell counts were unremarkable for most patients; one patient who had HBV-related liver cirrhosis showed moderate thrombocytopenia with a platelet count of $66,000 / \mu \mathrm{L}$ at the time of PEL diagnosis (Table 2).

\section{Immunophenotype and EBV status}

A representative case of PEL with a characteristic cytomorphology and immunohistochemical staining pattern for LANA1 is shown in Fig. 1. The results of immunophenotypic 
Table 2. Patient characteristics of the entire cohort and subgroups based on HHV8 positivity ${ }^{\text {a) }}$

\begin{tabular}{|c|c|c|c|c|}
\hline Characteristic & $\begin{array}{c}\text { Entire } \\
\text { cohort }(n=17)\end{array}$ & $\begin{array}{l}\text { PEL } \\
(n=6)\end{array}$ & $\begin{array}{l}\text { HHV8-unrelated } \\
\text { BCBL }(n=6)\end{array}$ & $\begin{array}{l}\text { HHV8-unknown } \\
\text { BCBL }(n=5)\end{array}$ \\
\hline Age at diagnosis (yr) & $73(39-87)$ & $68.5(59-87)$ & $76(59-83)$ & $73(39-86)$ \\
\hline \multicolumn{5}{|l|}{ Sex } \\
\hline Male & $11 / 17(64.7)$ & $5 / 6(83.3)$ & $5 / 6(83.3)$ & $1 / 5(20.0)$ \\
\hline Female & $6 / 17(35.3)$ & $1 / 6(16.7)$ & $1 / 6(16.7)$ & $4 / 5(80.0)$ \\
\hline \multicolumn{5}{|l|}{ Site of lymphoma involvement } \\
\hline Pleural cavity & $15 / 17(88.2)$ & $6 / 6(100)$ & $4 / 6(66.7)$ & $5 / 5(100)$ \\
\hline Pericardial cavity & $4 / 17(23.5)$ & $1 / 6(16.7)$ & $3 / 6(50.0)$ & $0 / 5(0)$ \\
\hline Peritoneal cavity & $4 / 17(23.5)$ & $1 / 6(16.7)$ & $1 / 6(16.7)$ & $2 / 5(40.0)$ \\
\hline Extra-cavitary lesion $^{\text {b) }}$ & 2/17 (11.8) & $1 / 6(16.7)$ & $0 / 6(0)$ & $1 / 5(20.0)$ \\
\hline \multicolumn{5}{|l|}{ ECOG PS at diagnosis } \\
\hline $0-1$ & $10 / 17(58.8)$ & $3 / 6(50.0)$ & $3 / 6(50.0)$ & $4 / 5(80.0)$ \\
\hline $2-4$ & $7 / 17(41.2)$ & $3 / 6(50.0)$ & $3 / 6(50.0)$ & $1 / 5(20.0)$ \\
\hline \multicolumn{5}{|l|}{ IPI risk group } \\
\hline Low $(0-1)$ & $3 / 16(18.8)$ & $2 / 5(40.0)$ & $1 / 6(16.7)$ & $0 / 5(0)$ \\
\hline Low-intermediate (2) & $1 / 16(6.2)$ & $0 / 5(0)$ & $0 / 6(0)$ & $1 / 5(20.0)$ \\
\hline High-intermediate (3) & $4 / 16(25.0)$ & $1 / 5(20.0)$ & $2 / 6(33.3)$ & $1 / 5(20.0)$ \\
\hline High (4-5) & $8 / 16(50.0)$ & $2 / 5(40.0)$ & $3 / 6(50.0)$ & $3 / 5(60.0)$ \\
\hline \multicolumn{5}{|l|}{ Comorbidity } \\
\hline HIV & $1 / 17(5.9)$ & $1 / 6(16.7)$ & $0 / 6(0)$ & $0 / 5(0)$ \\
\hline $\mathrm{HBV}$ & 2/17 (11.8) & $1 / 6(16.7)$ & $0 / 6(0)$ & $1 / 5(20.0)$ \\
\hline $\mathrm{HCV}$ & $1 / 17(5.9)$ & $0 / 6(0)$ & $1 / 6(16.7)$ & $0 / 5(0)$ \\
\hline Co-occurring malignancy & $\left.1 / 17(5.9)^{\mathrm{c}}\right)$ & $1 / 6(16.7)^{\mathrm{c})}$ & $0 / 6(0)$ & $0 / 5(0)$ \\
\hline \multicolumn{5}{|l|}{ Laboratory profile at diagnosis } \\
\hline $\mathrm{WBC} \geq 10,000 / \mu \mathrm{L}$ & $3 / 17(17.6)$ & $1 / 6(16.7)$ & $0 / 6(0)$ & $2 / 5(40.0)$ \\
\hline Hemoglobin $<10 \mathrm{~g} / \mathrm{dL}$ & $4 / 17(23.5)$ & $1 / 6(16.7)$ & $2 / 6(33.3)$ & $1 / 5(20.0)$ \\
\hline Platelet $<\left(130 \times 10^{3}\right) / \mu \mathrm{L}$ & $1 / 17(5.9)$ & $1 / 6(16.7)$ & $0 / 6(0)$ & $0 / 5(0)$ \\
\hline Serum albumin $<3.5 \mathrm{~g} / \mathrm{dL}$ & $12 / 17(70.6)$ & $5 / 6(83.3)$ & $5 / 6(83.3)$ & $2 / 5(40.0)$ \\
\hline Serum creatinine $\geq 1.5 \mathrm{mg} / \mathrm{dL}$ & $3 / 17(17.6)$ & $1 / 6(16.7)$ & $1 / 6(16.7)$ & $1 / 5(20.0)$ \\
\hline LDH above normal & $13 / 16(81.2)$ & $4 / 6(66.7)$ & $4 / 5(80.0)$ & $5 / 5(100)$ \\
\hline$\beta 2$-microglobulin $\geq 3.5 \mathrm{mg} / \mathrm{dL}$ & $5 / 10(50.0)$ & $2 / 4(50.0)$ & $2 / 5(40.0)$ & $1 / 1(100)$ \\
\hline C-reactive protein $\geq 0.8 \mathrm{mg} / \mathrm{dL}$ & $17 / 17(100)$ & $6 / 6(100)$ & $6 / 6(100)$ & $5 / 5(100)$ \\
\hline
\end{tabular}

Values are presented as median (range) or number (\%). HHV8, human herpesvirus 8; PEL, primary effusion lymphoma; BCBL, body cavity-based lymphoma; ECOG PS, Eastern Cooperative Oncology Group performance status; IPI, international prognostic index; $\mathrm{HIV}$, human immunodeficiency virus; $\mathrm{HBV}$, hepatitis B virus; $\mathrm{HCV}$, hepatitis $\mathrm{C}$ virus; $\mathrm{WBC}$, white blood cell; $\mathrm{LDH}$, lactate dehydrogenase. ${ }^{\mathrm{a}}$ The proportions are calculated as the frequency divided by the total number of evaluable patients for each characteristic, ${ }^{b}$ Extra-cavitary lesions were present in one patient with PEL who had both bone-marrow and cervical lymph-node lesions and one patient with HHV8-unknown BCBL who had a lung lesion, ${ }^{c}$ Lung cancer.

analysis and in situ hybridization for EBER are shown in Fig. 2. All six patients with PEL had diffuse and strong positivity for LANA1. Three of the six patients (50\%) with PEL were positive for EBER, whereas all but one of the patients (83.3\%) with HHV8-unrelated BCBL were negative for EBER. Of the five patients with PEL who were tested for CD20 expression, one was weakly positive ( $20 \%$ ); that patient also displayed CD79a expression with focal positivity (case 3). In contrast, all six patients with HHV8-unrelated BCBL were positive for CD20 expression. In the reports in the literature, lymphocyte activation markers CD30 and CD38, pan-leukocyte marker CD45, and plasma cell differentiation marker CD138 are all commonly expressed in patients with PEL [16]. Results for CD30, CD38, CD45, and CD138 were positive in four of five (80\%), two of two (100\%), one of three (33.3\%), and four of five $(80 \%)$ patients with PEL who were tested for those markers in our study, respectively. In contrast, results for CD30 and CD138 were negative in three of three $(100 \%)$ 


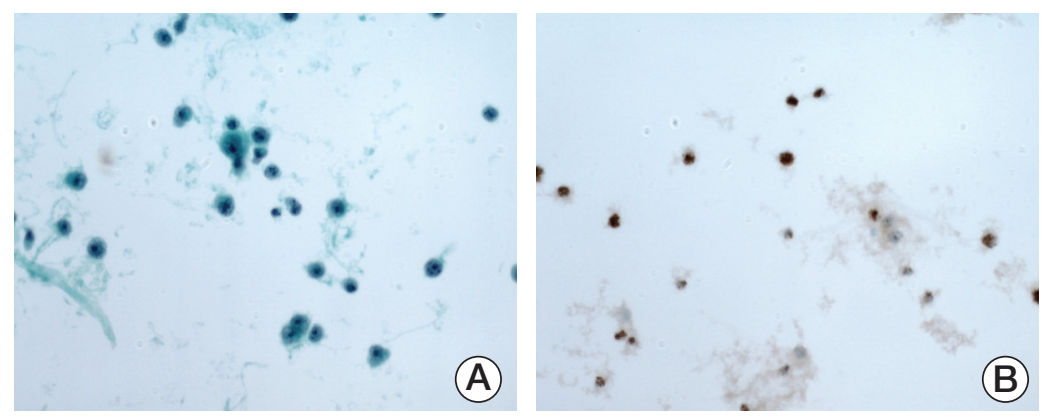

Fig. 1. Lymphoma cells with positive nuclear staining for latency-associated nuclear antigen 1 in the ascitic fluid of a representative primary effusion lymphoma patient. (A) Ascites smear exhibits individually scattered tumor cells with an immunoblastic or plasmablastic cytomorphology. (B) Immunohistochemistry using an anti-human herpesvirus 8 antibody shows dark brown nuclear staining of tumor cells.

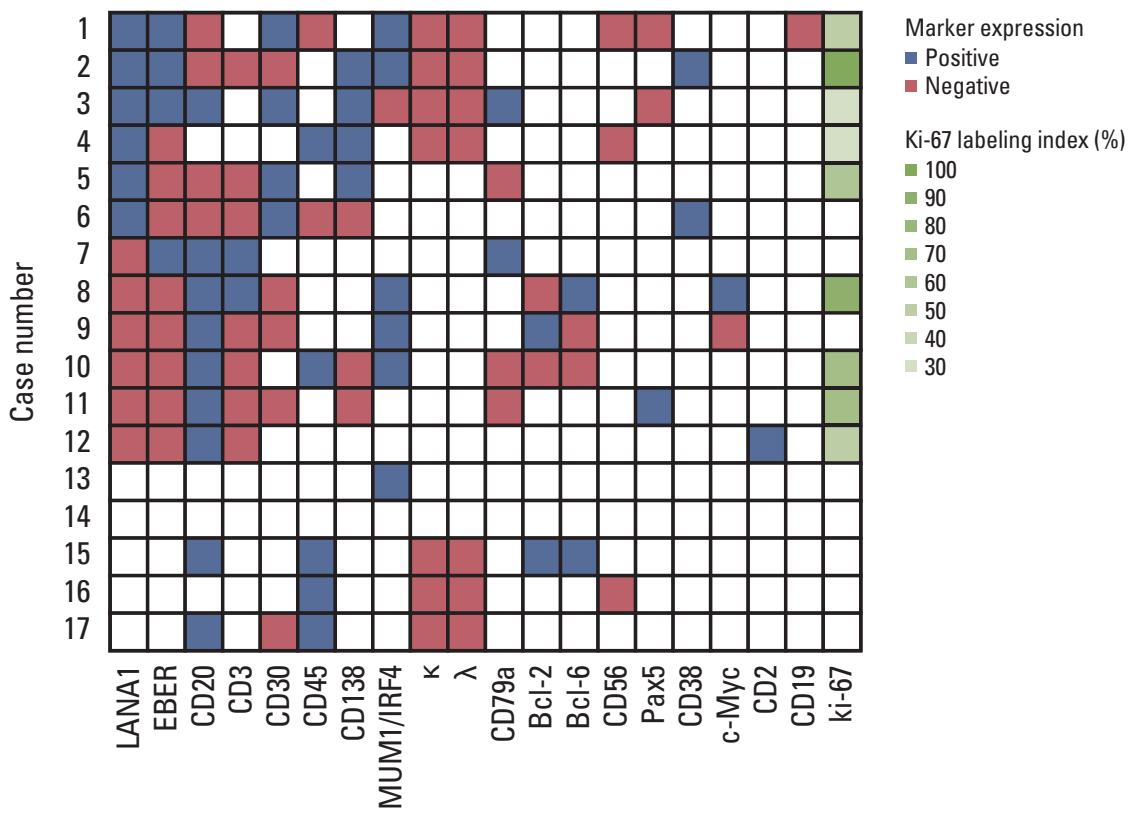

Fig. 2. Immunophenotypic analysis and in situ hybridization for Epstein-Barr virus-encoded small RNA (EBER). The cases are presented in the same order as in Table 1. Latency-associated nuclear antigen 1 (LANA1) and EBER are shown in the first and second columns, respectively, while the remaining markers are arranged in descending order of the number of cases in which they were analyzed. All markers that were stained in at least one case are shown. Blank tiles indicate that assays were not performed or data were not available. MUM1, multiple myeloma oncogene 1; IRF4, interferon regulatory factor $4 ; \kappa$, immunoglobulin $\kappa$ light chain; $\lambda$, immunoglobulin $\lambda$ light chain.

and two of two $(100 \%)$ patients with HHV8-unrelated BCBL who were tested for those markers, respectively. Results for multiple myeloma oncogene 1 / interferon regulatory factor 4 were positive in six of seven patients tested $(85.7 \%)$, including two patients with PEL, three patients with HHV8-unrelated BCBL, and one patient with HHV8-unknown BCBL.
Results for immunoglobulin $\kappa$ and $\lambda$ light chains were negative in all seven of the patients tested. Among the five patients with PEL and four patients with HHV8-unrelated BCBL who were tested for the fraction of Ki-67-positive tumor cells (Ki-67 labeling index), three with PEL had a Ki-67 labeling index $\leq 40 \%$ (cases 3 and 4,30\%; case 1,40\%), indicating a 
Table 3. First-line chemotherapy regimens

\begin{tabular}{lcccc} 
Regimen & $\begin{array}{c}\text { Entire } \\
\text { cohort }(\mathbf{n}=\mathbf{1 7})\end{array}$ & $\begin{array}{c}\text { PEL } \\
(\mathbf{n}=\mathbf{6})\end{array}$ & $\begin{array}{c}\text { HHV8-unrelated } \\
\text { BCBL }(\mathbf{n}=6)\end{array}$ & $\begin{array}{c}\text { HHV8-unknown } \\
\text { BCBL }(\mathbf{n}=5)\end{array}$ \\
CHOP & $5(29.4)$ & $3(50.0)$ & 0 & $2(40.0)$ \\
R-CHOP & $5(29.4)$ & 0 & $4(66.7)$ & $1(20.0)$ \\
CVP & $1(5.9)$ & 0 & 0 & $1(20.0)$ \\
R-CVP & $2(11.8)$ & 0 & $1(16.7)$ & $1(20.0)$ \\
HD-MTX & $1(5.9)$ & 0 & 0 & 0 \\
No chemotherapy & $3(17.6)$ & $3(50.0)$ & $1(16.7)$ & 0 \\
\hline
\end{tabular}

Values are presented as number (\%). PEL, primary effusion lymphoma; HHV8, human herpesvirus 8; BCBL, body cavitybased lymphoma; $\mathrm{CHOP}$, cyclophosphamide, doxorubicin, vincristine, and prednisolone; $\mathrm{R}-\mathrm{CHOP}$, rituximab plus $\mathrm{CHOP}$; CVP, cyclophosphamide, vincristine, and prednisolone; R-CVP, rituximab plus CVP; HD-MTX, high-dose methotrexate.

Table 4. Comparison of clinical characteristics and outcome of classic PEL cases between the historical controls ${ }^{\mathrm{a})}$ and the present cohort

\begin{tabular}{lcccccc} 
Characteristic & $\begin{array}{c}\text { Nador } \\
\text { et al. [4] }\end{array}$ & $\begin{array}{c}\text { Boulanger } \\
\text { et al. [5] }\end{array}$ & $\begin{array}{c}\text { Simonelli } \\
\text { et al. [6] }\end{array}$ & $\begin{array}{c}\text { Boulanger } \\
\text { et al. [7] }\end{array}$ & $\begin{array}{c}\text { Guillet } \\
\text { et al. [8] }\end{array}$ & $\begin{array}{c}\text { Present } \\
\text { cohort }\end{array}$ \\
No. of cases & 15 & 12 & 11 & 28 & 34 & 6 \\
Male-to-female ratio & $15: 0$ & $12: 0$ & $10: 1$ & $27: 1$ & $31: 3$ & $5: 1$ \\
\hline Age, median (range, yr) & $44(31-85)$ & $43.5(33-66)$ & $41(26-58)$ & $44(33-78)$ & $45(40-54)$ & $68.5(59-87)$ \\
HIV positivity rate $(\%)$ & 87 & 100 & 100 & 100 & 100 & 17 \\
Median survival & $5 \mathrm{mo}$ & $5.6 \mathrm{mo}$ & $6 \mathrm{mo}$ & $6.2 \mathrm{mo}$ & $10.2 \mathrm{mo}$ & $4.1 \mathrm{yr}$ \\
\hline
\end{tabular}

PEL, primary effusion lymphoma; HIV, human immunodeficiency virus. ${ }^{\text {a) }}$ The five largest case series of PEL reported thus far (to our knowledge) were used for comparison.

low proliferative activity and indolent disease, whereas all four with HHV8-unrelated BCBL had a Ki-67 labeling index $\geq 50 \%$ (case 8, 95\%; cases 10 and 11, 90\%; case 12,50\%). Immunophenotype data were limited for the patients with HHV8-unknown BCBL; CD20 and CD45 were expressed in all two (100\%) and three (100\%) of the patients with HHV8unknown BCBL who were tested, respectively.

\section{Treatment and response}

Fourteen patients were treated with cytotoxic chemotherapy. Three of the patients with PEL did not receive any chemotherapy because of old age (cases 4 and 5) or an unknown reason (case 3). The first-line treatments are summarized in Table 3. Most patients received combination chemotherapy of cyclophosphamide, doxorubicin, vincristine, and prednisolone $(\mathrm{CHOP})$ with $(n=5)$ or without $(n=5)$ rituximab. All three patients with PEL who were treated with chemotherapy were negative for $\mathrm{CD} 20$ and received $\mathrm{CHOP}$ alone as a first-line treatment. All but one of the patients with HHV8unrelated BCBL (all positive for CD20) received rituximab- containing regimens such as rituximab plus $\mathrm{CHOP}$ (R-CHOP, $\mathrm{n}=4$ ) or rituximab plus cyclophosphamide, vincristine, and prednisolone ( $\mathrm{R}-\mathrm{CVP}, \mathrm{n}=1$ ). The remaining patient with HHV8-unrelated BCBL, who presented with a malignant effusion involving only the vitreous cavity of the eye, was treated with high-dose methotrexate, which resulted in complete remission (CR). The first-line regimens were more heterogeneous in the HHV8-unknown BCBL cohort (Table 3).

We had response information for 12 of the 14 patients that received cytotoxic chemotherapy. Overall, nine patients (75\%) achieved CR and three patients (25\%) achieved partial remission (PR) after the first-line treatment. R-CHOP resulted in CR in all five of the patients who received it (four with HHV8-unrelated BCBL and one with HHV8-unknown $\mathrm{BCBL})$. CHOP alone resulted in one PR and one $\mathrm{CR}$ in the PEL cohort and two CRs in the HHV8-unknown BCBL cohort. Two patients with HHV8-unknown BCBL who were treated with cyclophosphamide, vincristine, and prednisolone (CVP)-based regimens achieved PR (cases 14 and 15). One patient with PEL who received CHOP and achieved CR experienced relapse of the lymphoma at 28 months after ini- 
tial diagnosis and was subsequently treated with ifosfamide, carboplatin, and etoposide (ICE) combination chemotherapy followed by autologous hematopoietic stem cell transplantation (autoHSCT), which resulted in PR (case 6).

\section{Survival}

The PFS and OS of each patient are described in Table 1. During a median follow-up period of 1.4 years (range, 0.2 to 14.4 years), one patient with PEL (5.9\% overall) experienced disease progression, and three patients (17.6\% overall) died, one each with PEL (case 4), HHV8-unrelated BCBL (case 7), and HHV8-unknown BCBL (case 14). The cause of death was unknown for the patient with PEL and pneumonia for the other two patients.

The patient with PEL that showed the lowest tumor cell growth fraction (case 4 ) survived for $>4$ years without any chemotherapy. Another patient with PEL who had HIV infection (case 3 ) survived for $>1$ year without chemotherapy. The patient with PEL that relapsed 28 months after CR lived for $>12$ years thereafter with salvage ICE chemotherapy and autoHSCT (case 6). In the PEL, HHV8-unrelated BCBL, and HHV8-unknown BCBL cohorts, the median PFS was 3.2 years, 5.5 years, and not reached, respectively, and the median OS was 4.1 years, 5.5 years, and not reached, respectively.

\section{Discussion}

We described 17 patients with BCBL diagnosed in South Korea with a focus on clinicopathologic characteristics and outcomes. We divided the patients into three categories based on the results of immunocytochemical staining for LANA1, which is currently the standard assay to detect HHV8 infection in lymphoma cells, an essential requirement for a diagnosis of PEL [1]. We found a remarkable lack of association between immunocompromising conditions and PEL. Only one of six patients with PEL was HIV seropositive, and no other patient had a prior history of organ transplantation, immunosuppressant treatment, or cytotoxic chemotherapy. Therefore, five of the six patients with PEL in our series were ostensibly immunocompetent. By contrast, more than $90 \%$ of patients with PEL described in the literature are HIV seropositive, mostly with severely decreased circulating CD4-positive T cell counts (Table 4) [4-8]. That remarkable difference in the rate of HIV seropositivity among patients with PEL between our series and the patients described in the literature-most of whom are Caucasians from regions with moderate-to-high HIV prevalence-is in line with the fact that South Korea has one of the lowest HIV burdens worldwide, with an HIV seroprevalence of only $0.003 \%$ $0.01 \%$ [21].

Patients with PEL in our study had a favorable prognosis with a median OS of 4.1 years, whereas those in previous studies consistently had poor outcomes, with a median OS of around 6 months (Table 4) [4-8]. Most patients in the literature with PEL and HIV infection had severe immunodeficiency at the time of PEL diagnosis, with more than $60 \%$ having a prior history of AIDS-related illness and a CD4-positive T cell count of $<200 / \mu \mathrm{L}$ [4-8]. Four out of eight deaths in a previous series of patients with HIV infection and PEL were due to causes unrelated to PEL, such as sepsis or hemoptysis [5]. In addition, there have been several reports in which patients with HIV infection and PEL were successfully treated with antiretroviral therapy alone $[6,22,23]$. In the present cohort, two patients with the lowest Ki-67 labeling index (30\%) survived for 14 and 49 months each without having had chemotherapy at the end of follow-up, which is in contrast to the PEL patients in the literature who mostly demonstrated a high proliferation index [24]. Taken together, our findings suggest that the poor outcomes observed in historical PEL cases are largely due to HIV-related complications, such as opportunistic infections and malignancies, rather than to PEL. In that case, watchful waiting with only supportive measures such as drainage of the effusion would be appropriate for the management of chemotherapy-intolerant, HIV-seronegative patients with PEL. In particular, PEL with low proliferation activity might represent an indolent subset that patients can survive for a long time, suggesting the potential value of Ki-67 immunostaining for the prognostic stratification of PEL. Given the small size and the retrospective nature of our case series, future prospective studies are needed to confirm those hypotheses.

Although the detection of HHV8 infection in neoplastic cells is essential to make a diagnosis of PEL as per the WHO criteria [3], we found that five out of 17 patients with BCBL were not tested for HHV8 positivity, and the majority of patients with HHV8-unrelated or HHV8-unknown BCBL were mislabeled as having PEL in their medical records or pathology reports (data not shown). The frequent misdiagnosis of HHV8-unrelated BCBL as PEL is due in part to the lack of a specific subcategory describing HHV8-unrelated BCBL in the current NHL classification despite its unique clinical features [17]. For that reason, there are many different terms for HHV8-unrelated BCBL in the literature, such as HHV8unrelated PEL-like lymphoma [15,18], HHV8-negative malignant effusion lymphoma [14], and HHV8-negative effusion-based lymphoma [17], causing difficulties in clarifying their clinical entity. Discrimination between PEL and HHV8-unrelated BCBL is crucial, because patients with HHV8-unrelated BCBL have a high expression rate $(>80 \%)$ 
of pan-B-cell markers including CD20, whereas those with PEL do not [16-18], which means that rituximab should have therapeutic value for HHV8-unrelated BCBL but not for PEL. Indeed, in our study, all four patients with HHV8-unrelated BCBL who were treated with R-CHOP achieved CR.

In our study as well as in the literature, patients with PEL, but not those with HHV8-unrelated BCBL, were frequently positive for $\mathrm{CD} 30$ expression $[7,8,16]$. The high rate of $\mathrm{CD} 30$ expression in PEL is reminiscent of other CD30-expressing lymphomas such as anaplastic large cell lymphoma, Hodgkin lymphoma, and a subset of diffuse large B-cell lymphoma, for which brentuximab vedotin, an anti-CD30 antibody conjugated to the anti-microtubule agent monomethyl auristatin E, has shown antitumor activity [25-28]. In a recent preclinical study, brentuximab vedotin demonstrated a promising antitumor effect on PEL cell lines and xenograft mice, urging further investigation in clinical trials [29]. The findings of our study suggest that physicians should give more attention to precise discrimination between HHV8-unrelated BCBL and PEL for optimal management and clinical trial design.

Our study has several limitations. First, the small number of cases and the incompleteness of the data preclude drawing firm conclusions in many aspects. For instance, we could not determine whether the tumor cell immunophenotype of PEL differed between immunocompetent patients and immunocompromised patients. Although our results suggest that PEL may have more favorable outcomes in patients without HIV infection than in those with HIV infection, we did not test that hypothesis explicitly, which will require a prospective study with a larger cohort. Second, as this study is a medical record-based retrospective study with no central pathology review conducted, the determination of LANA1 immunostaining positivity was not standardized across participating institutions. Third, most of our analyses were descriptive in nature, with no formal statistical testing. Nonetheless, PEL is so rare that, even worldwide, the largest consecutive series of classic PEL cases included only 34 patients, and that of HHV8-unrelated BCBL cases is even smaller $[8,17]$. To our knowledge, our series represents the largest collection of consecutive Asian patients with PEL or HHV8unrelated or HHV8-unknown BCBL.

In conclusion, in a consecutive series of patients with $\mathrm{BCBL}$ in a country with low HIV burden, most patients with PEL were HIV seronegative and immunocompetent. Those patients, especially those with low proliferation activity of neoplastic cells, had an indolent disease course and long survival even with supportive care only. Our findings raise a testable hypothesis that watchful waiting is a reasonable approach when managing HIV-seronegative patients with PEL who may not tolerate chemotherapy or whose neoplastic cells show low proliferative activity. The Ki-67 labeling index might serve as a marker for the prognostic stratification of PEL. HHV8-unrelated BCBL was successfully treated with rituximab-containing chemotherapy, which corroborates the findings of previous studies [14-18]. We also found that physicians often fail to distinguish between HHV8-unrelated BCBL and PEL despite the important therapeutic implications. The upcoming revisions of the WHO classification of lymphoid neoplasms should be based on a thorough discussion of the most proper categorization of HHV8-unrelated BCBL. Several emerging hypotheses generated from the present study should be confirmed in future prospective studies based on larger cohorts.

\section{Conflicts of Interest}

Conflict of interest relevant to this article was not reported.

\section{Acknowledgments}

This work was supported by a grant from the National Research Foundation of Korea for the Cellular Heterogeneity Research Center (NRF-2016R1A5A1011974), funded by the Ministry of Science, ICT \& Future planning, Republic of Korea. The authors thank the Consortium for Improving Survival of Lymphoma for their efforts in coordinating the study. This manuscript has been edited by native English-speaking experts associated with BioScience Writers LLC, Houston, TX, USA.

\section{Author Details}

${ }^{1}$ Department of Internal Medicine, Seoul National University Hospital, Seoul, '2Department of Pathology, Samsung Medical Center, Seoul, ${ }^{3}$ Department of Internal Medicine, Dong-A University College of Medicine, Busan, ${ }^{4}$ Department of Oncology, Asan Medical Center, Seoul, ${ }^{5}$ Department of Internal Medicine, Seoul National University Bundang Hospital, Seongnam, ${ }^{6}$ Department of Internal Medicine, Yonsei University College of Medicine, Seoul, ${ }^{7}$ Department of Internal Medicine, Korea University Anam Hospital, Seoul, ${ }^{8}$ Department of Internal Medicine, Pusan National University Hospital, Busan, ${ }^{9}$ Department of Medicine, Samsung Medical Center, Seoul, ${ }^{10}$ Department of Internal Medicine, Soonchunhyang University Seoul Hospital, Seoul, Korea 


\section{References}

1. Chen YB, Rahemtullah A, Hochberg E. Primary effusion lymphoma. Oncologist. 2007;12:569-76.

2. Cesarman E, Chang Y, Moore PS, Said JW, Knowles DM. Kaposi's sarcoma-associated herpesvirus-like DNA sequences in AIDS-related body-cavity-based lymphomas. N Engl J Med. 1995;332:1186-91.

3. Swerdlow SH, Campo E, Pileri SA, Harris NL, Stein H, Siebert $\mathrm{R}$, et al. The 2016 revision of the World Health Organization classification of lymphoid neoplasms. Blood. 2016;127:237590.

4. Nador RG, Cesarman E, Chadburn A, Dawson DB, Ansari $M Q$, Sald J, et al. Primary effusion lymphoma: a distinct clinicopathologic entity associated with the Kaposi's sarcomaassociated herpes virus. Blood. 1996;88:645-56.

5. Boulanger E, Agbalika F, Maarek O, Daniel MT, Grollet L, Molina JM, et al. A clinical, molecular and cytogenetic study of 12 cases of human herpesvirus 8 associated primary effusion lymphoma in HIV-infected patients. Hematol J. 2001;2: 172-9.

6. Simonelli C, Spina M, Cinelli R, Talamini R, Tedeschi R, Gloghini A, et al. Clinical features and outcome of primary effusion lymphoma in HIV-infected patients: a single-institution study. J Clin Oncol. 2003;21:3948-54.

7. Boulanger E, Gerard L, Gabarre J, Molina JM, Rapp C, Abino JF, et al. Prognostic factors and outcome of human herpesvirus 8 -associated primary effusion lymphoma in patients with AIDS. J Clin Oncol. 2005;23:4372-80.

8. Guillet S, Gerard L, Meignin V, Agbalika F, Cuccini W, Denis $\mathrm{B}$, et al. Classic and extracavitary primary effusion lymphoma in $51 \mathrm{HIV}$-infected patients from a single institution. Am J Hematol. 2016;91:233-7.

9. Said JW, Tasaka T, Takeuchi S, Asou H, de Vos S, Cesarman E, et al. Primary effusion lymphoma in women: report of two cases of Kaposi's sarcoma herpes virus-associated effusionbased lymphoma in human immunodeficiency virus-negative women. Blood. 1996;88:3124-8.

10. Jones D, Ballestas ME, Kaye KM, Gulizia JM, Winters GL, Fletcher J, et al. Primary-effusion lymphoma and Kaposi's sarcoma in a cardiac-transplant recipient. N Engl J Med. 1998;339: 444-9.

11. Boulanger E, Afonso PV, Yahiaoui Y, Adle-Biassette H, Gabare J, Agbalika F. Human herpesvirus-8 (HHV-8)-associated primary effusion lymphoma in two renal transplant recipients receiving rapamycin. Am J Transplant. 2008;8:707-10.

12. Hermine O, Michel M, Buzyn-Veil A, Gessain A. Body-cavitybased lymphoma in an HIV-seronegative patient without Kaposi's sarcoma-associated herpesvirus-like DNA sequences. N Engl J Med. 1996;334:272-3.

13. Rodriguez J, Romaguera JE, Katz RL, Said J, Cabanillas F. Primary effusion lymphoma in an HIV-negative patient with no serologic evidence of Kaposi's sarcoma virus. Leuk Lymphoma. 2001;41:185-9.

14. Matsumoto Y, Nomura K, Ueda K, Satoh K, Yasuda N, Taki
T, et al. Human herpesvirus 8-negative malignant effusion lymphoma: a distinct clinical entity and successful treatment with rituximab. Leuk Lymphoma. 2005;46:415-9.

15. Shin J, Lee JO, Choe JY, Bang SM, Lee JS. Human herpesvirus 8-unrelated primary effusion lymphoma-like lymphoma in an elderly Korean patient with a good response to rituximab plus cyclophosphamide, doxorubicin, vincristine, and prednisolone. Cancer Res Treat. 2017;49:274-8.

16. Kobayashi Y, Kamitsuji Y, Kuroda J, Tsunoda S, Uoshima N, Kimura S, et al. Comparison of human herpes virus 8 related primary effusion lymphoma with human herpes virus 8 unrelated primary effusion lymphoma-like lymphoma on the basis of HIV: report of 2 cases and review of 212 cases in the literature. Acta Haematol. 2007;117:132-44.

17. Alexanian S, Said J, Lones M, Pullarkat ST. KSHV / HHV8-negative effusion-based lymphoma, a distinct entity associated with fluid overload states. Am J Surg Pathol. 2013;37:241-9.

18. Wu W, Youm W, Rezk SA, Zhao X. Human herpesvirus 8unrelated primary effusion lymphoma-like lymphoma: report of a rare case and review of 54 cases in the literature. Am J Clin Pathol. 2013;140:258-73.

19. Hall JC, Hall BJ, Cockerell CJ. HIV / AIDS in the post-HAART era: manifestations, treatment, and epidemiology. Shelton, CT: People's Medical Publishing House, USA; 2011.

20. Cheson BD, Pfistner B, Juweid ME, Gascoyne RD, Specht L, Horning SJ, et al. Revised response criteria for malignant lymphoma. J Clin Oncol. 2007;25:579-86.

21. Choe KW. Epidemiology of HIV/ AIDS: current status, trend and prospect. J Korean Med Assoc. 2007;50:296-302.

22. Oksenhendler E, Clauvel JP, Jouveshomme S, Davi F, Mansour G. Complete remission of a primary effusion lymphoma with antiretroviral therapy. Am J Hematol. 1998;57:266.

23. Hocqueloux L, Agbalika F, Oksenhendler E, Molina JM. Longterm remission of an AIDS-related primary effusion lymphoma with antiviral therapy. AIDS. 2001;15:280-2.

24. Carbone A, Gloghini A, Bontempo D, Monini P, Tirelli U, Volpe $\mathrm{R}$, et al. Proliferation in HHV-8-positive primary effusion lymphomas is associated with expression of HHV-8 cyclin but independent of p27(kip1). Am J Pathol. 2000;156: 1209-15.

25. Pro B, Advani R, Brice P, Bartlett NL, Rosenblatt JD, Illidge T, et al. Five-year results of brentuximab vedotin in patients with relapsed or refractory systemic anaplastic large cell lymphoma. Blood. 2017;130:2709-17.

26. Younes A, Gopal AK, Smith SE, Ansell SM, Rosenblatt JD, Savage KJ, et al. Results of a pivotal phase II study of brentuximab vedotin for patients with relapsed or refractory Hodgkin's lymphoma. J Clin Oncol. 2012;30:2183-9.

27. Connors JM, Jurczak W, Straus DJ, Ansell SM, Kim WS, Gallamini A, et al. Brentuximab vedotin with chemotherapy for stage III or IV Hodgkin's lymphoma. N Engl J Med. 2018;378: 331-44.

28. Jacobsen ED, Sharman JP, Oki Y, Advani RH, Winter JN, Bello 
$\mathrm{CM}$, et al. Brentuximab vedotin demonstrates objective responses in a phase 2 study of relapsed / refractory DLBCL with variable CD30 expression. Blood. 2015;125:1394-402.

29. Bhatt S, Ashlock BM, Natkunam Y, Sujoy V, Chapman JR,
Ramos JC, et al. CD30 targeting with brentuximab vedotin: a novel therapeutic approach to primary effusion lymphoma. Blood. 2013;122:1233-42. 